\title{
Population Growth Models of Forest Trees for Conservation Management: Case of Teak (Tectona Grandis) Forest in Begal, East Java, Indonesia
}

\author{
Novriana Sumarti*, Mharta A. Wardana, Nuning Nuraini \\ Faculty of Mathematics and Natural Sciences, Institut Teknologi Bandung, Bandung, Indonesia
}

\section{ARTICLE INFO}

Article history:

Received February 10, 2017

Received in revised form December 15, 2017

Accepted January 17, 2018

\section{KEYWORDS:}

Forest Conservation, Forest Management, Mathematical Model, Prediction

\begin{abstract}
Based on 2010 FAO report, teak forest and plantation in Indonesia covers 1,269 million hectares or 7 per mill of total area of Indonesia. It can be found dominantly in Central and East Java. PT Perhutani, Indonesia has responsible for management of the government owned forests in the islands of Java and Madura. Based on 2007 data, the teak wood production is $517,627 \mathrm{~m}^{3}$ and the highest percentage, which is $37 \%$ of total production, is coming from East Java. In this paper, we develop growth population models using Leslie Matrix and Markov Chain in order to predict the future condition based on the current condition. The models are implemented into data from Teak Forest in Begal, East Java, that covers 2,052.8 hectares and consists of 114 sites. The result from the first model using Leslie Matrix shows that it needs 16 years from year 2011 that the sustainable condition of the forest can be achieved. The result from the implementation of the second model using Markov Chain into the existing data shows that the condition of the teak forest can be classified as quite critical because the good condition part based on its density of the early age group ( 0 - 4 years) has potential to become the worst condition before its harvest time.
\end{abstract}

\section{Introduction}

Teak tree (Tectona grandis) is a tropical hardwood that becomes the most popular choice for furniture, exterior construction, carving, boat building, and others, due to its durability and water resistance. Native habitants of this tree are in south and Southeast Asia, mainly India, Sri Lanka, Indonesia, Malaysia, Thailand, Myanmar, and Bangladesh (US Agriculture Dept 2007). It has been studied another good benefit on having teak forest in the area by through its dendroclimatic potential, which is the prediction of past climates by observing the tree-ring-width index chronologies of teak wood (Ram 2008) and (D'Arrigo 2011).

Researchers had been studying to find the suitable growth model of teak tree. Author in (Nunifu 1997) presented a preliminary study at assessing the growth and yield potential and developing provisional yield models for the management of teak in Northem Ghana. The diameter distribution, modelled by the threeparameter Weibull function, showed positive skewness

\footnotetext{
* Corresponding Author

E-mail Address: novriana@math.itb.ac.id
}

indicating there are more trees in smaller diameter classes. Initial planting spacing of 2 by $2 \mathrm{~m}$ could be reduced to accommodate initial mortality and to achieve optimum stocking levels in order to improve form and timber quality. This assessment resulted the potential for growing teak to acceptable timber size on good sites.

In (Tewari 2014), the state-space approach is used for modelling rates of change of three state-variables: dominant height, number of trees per hectare and stand basal area. The model includes a stand volume equation as an out-put function to estimate this variable at any point in time. The model is implemented into data from 22 teak sample plots at Karnataka State of Peninsular India.

Authors in (Shamaki 2016) discussed a model of height-diameter relationship on Teak tree and implemented into data from Nimbia Forest Reserve, Nigeria. The fitting methods being used are ChapmanRichards and Weibull. Chapman-Richards function produced the best goodness-of-fit statistics for Teak height-diameter modelling.

In this paper we consider a population growth model using Leslie Matrix, which is the matrix population models that useful in predicting population growth 
(Leslie 1948) and (Caswell 2001). This method had been used in many applications including in (Crouse 1987) and (Smith 1994). Authors in (Crouse 1987) proposed a modification of the original Leslie Matrix by adding the element of probability of individuals in a group of various ages will survive staying in the same group. The method is implemented into data of loggerhead turtle population in southeastern United States. This modified matrix is also used to develop the population growth model of teak trees population in this paper.

In (Smith 1994), the method is implemented into the fecundity (fertility) and mortality data of wild rabbit Oryctolagus cuniculus populations in England. Its population growth rate was shown to be slightly greater than unity in an average year. It means the populations is increasing, so there is a need to implement two kind of control policies; removing more adults than juveniles and vice versa, which are proposed to be implemented respectively at particular and different times.

Another population growth model considered in this paper is using Markov Chain, which is the matrix containing probabilities of transition between two consecutive events. In 1907, A. A. Markov began the study the type of chance where the outcome of a given experiment can affect the outcome of the next experiment, which is later called a Markov chain (Kemenny 1976) and (Sheldon 2010).

\section{Materials and Methods}

\subsection{Teak Forest}

Based on the Constitution of Republic of Indonesia Number 41 Year 1999 Regarding Forestry, forest areas are designated and or specified by the government to be maintained as permanent forests. Efforts in maintaining sustainable forest areas can be interpreted as to maintain the existence of permanent forest as the original state. In this state, the forest plants generally are dominated by old trees, so their strong roots and wide leaves' canopy can maximize the function of forests as one of the factors supporting the cycle of hydrology. From a long time ago, the good condition of hydrology cycle reflected on a stable climate for years up to about the end of 19th century. Nowadays, people's activities on forest tree looting or illegal logging had given bad impact not also on the climate change but also on an economic loss. In Indonesia, this loss suffered by PT Perhutani, the company owned by the government that has duty and authority to organize the planning and management of the exploitation and protection at Indonesian forests. Table 1 shows the economic loss due to a quite massive illegal logging at PT Perhutani's teak forest from 1999 to 2001. Many of the stolen trees were in old ages so the lack of these old trees in the

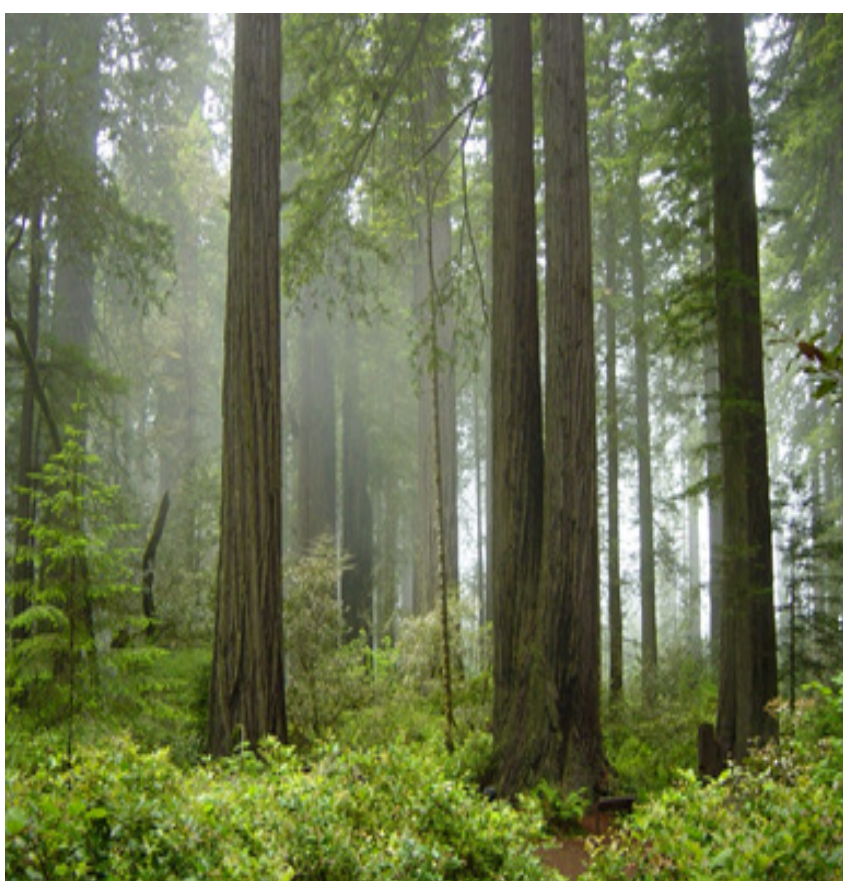

Figure 1. A picture sample of a teak forest (Redwood National Park wiki)

Table 1. Estimated economic loss

\begin{tabular}{lcc}
\hline Year & Number of stolen trees & $\begin{array}{c}\text { Economic loss } \\
\text { (rupiah) }\end{array}$ \\
\hline 1999 & $3,179,973$ & $55,851,584,000$ \\
2000 & $2,574,948$ & $569,757,232,000$ \\
2001 & $2,675,161$ & $613,924,367,000$ \\
\hline
\end{tabular}

forest could cause serious problems in maintaining its conversation.

Teak trees have age based stages and will be harvested after 60 years. The age classification of teak tree consist of classes Nursling/Young Plants (0-4), I (5-9), II (10 -19), III (20-29), IV (30-39), V (40-49), VI (50-59). In the period after class VI before harvesting, the tree is smoothed to turn off its cambium so the wood has high quality hardness. A part of forest area classified into the Nursling class can be interpreted as a void area where a severe looting activity had been occurred and induced deforestation in that area. This condition is undesirable and should be prevented by PT Perhutani. It is assumed that this area is allowed to exist only about one hectare of the total forest areas.

In order to improve wood quality and growth of individual high-quality trees, the management should do thinning and pruning not only in early stages but also often during later development of the plantation. PT Perhutani has used the Standard Density per hectare for Thinning (obtained from Buku Prosedur Kerja) that is shown in Table 2. 
Table 2. Standard density for teak tree thinning management

\begin{tabular}{|c|c|c|c|c|c|c|c|c|c|}
\hline Age & Density & Age & Density & Age & Density & Age & Density & Age & Density \\
\hline $0-4$ & 2045 & 16 & 667 & 28 & 387 & 40 & 269 & 52 & 201 \\
\hline 5 & 1769 & 17 & 634 & 29 & 373 & 41 & 263 & 53 & 197 \\
\hline 6 & 1617 & 18 & 600 & 30 & 359 & 42 & 256 & 54 & 194 \\
\hline 7 & 1466 & 19 & 567 & 31 & 348 & 43 & 251 & 55 & 190 \\
\hline 8 & 1314 & 20 & 524 & 32 & 338 & 44 & 245 & 56 & 187 \\
\hline 9 & 1163 & 21 & 513 & 33 & 328 & 45 & 240 & 57 & 180 \\
\hline 10 & 1012 & 22 & 492 & 34 & 319 & 46 & 234 & 58 & 176 \\
\hline 11 & 949 & 23 & 471 & 35 & 308 & 47 & 230 & 59 & 173 \\
\hline 12 & 887 & 24 & 450 & 36 & 300 & 48 & 225 & & \\
\hline 13 & 825 & 25 & 430 & 37 & 292 & 49 & 221 & & \\
\hline 14 & 763 & 26 & 416 & 38 & 284 & 50 & 208 & & \\
\hline 15 & 701 & 27 & 401 & 39 & 277 & 51 & 204 & & \\
\hline
\end{tabular}

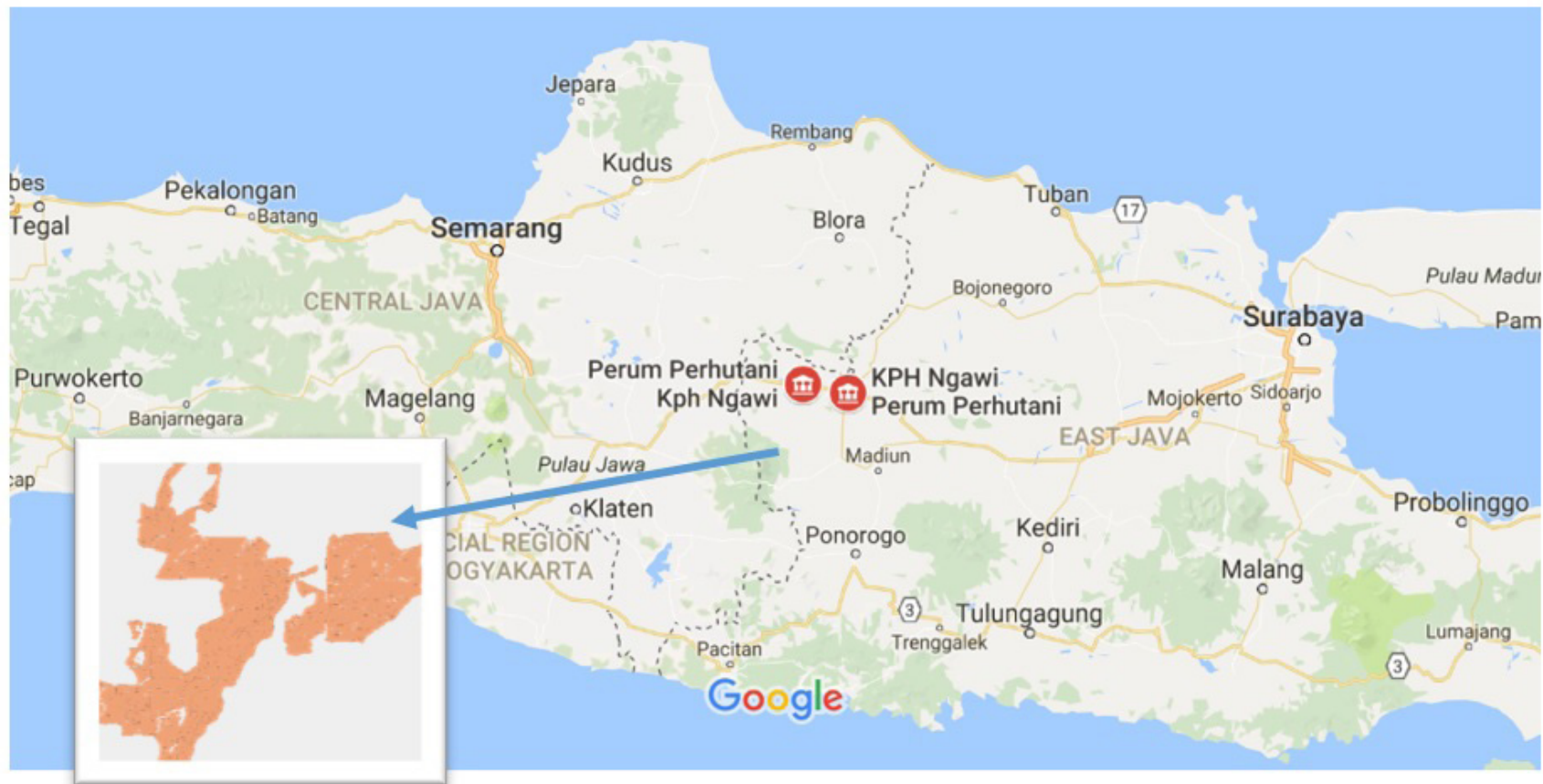

Figure 2. Map of KPH Ngawi PT Perhutani (Google Maps)

\subsection{Model of Growth Population}

\subsubsection{Leslie Matrix}

The growth model of teak trees population in a forest area will be derived based on modified Leslie Matrix that had been implemented into the population model of Loggerhead Turtle (Crouse 1987). The Leslie Model is powerful tool to determine the growth of a population as well as the age distribution within a population over time. The Leslie matrix population model is a discrete (or finite, i.e time goes in steps as opposed to continuously) and Age-Structured Population (construction of the model consider only age).

Let $m$ be the number of groups containing particular age stages in a population, $d_{i}$ be the group duration that is the age difference between the oldest and the youngest individuals in group $i, n(t)$ be a population vector containing $n_{1}(t), n_{2}(t), \ldots, n_{m}(t)$ representing the numbers of individuals in each group at time $t$, and $n(t+1)$ be a population vector in the next year.

$$
n(t+1)=A n(t)
$$

Where $A$ is the modified Leslie matrix, based on (Crouse et al. 1987).

$$
A=\left(\begin{array}{cccccc}
P_{1} & F_{1} & F_{2} & \cdots & F_{m-1} & F_{m} \\
G_{1} & P_{2} & 0 & \cdots & 0 & 0 \\
0 & G_{2} & \ddots & \cdots & \vdots & \vdots \\
\vdots & \uplus_{1} & \ddots & \ddots & \vdots & \vdots \\
0 & \cdots & \cdots & \ddots & P_{m-1} & 0 \\
0 & 0 & \cdots & \cdots & G_{m-1} & P_{m}
\end{array}\right)
$$


Note that $A$ is not tridiagonal matrix. The possible nonzero entries are only in the first row, the diagonal, and one place below diagonal. Fertility $F_{i}$ is the number of offspring of a group $i$ to $i+1$ in a unit of time $t$ that will survive to the next age stage group at time $t+1$. Survival $G_{i}$ is the probability that individuals in group $i$ at time $t$ will survive to group $i+1$ at time $t+1$. Survival $P_{i}$ is the probability that individuals in group $i$ at time $t$ will survive getting older but they are still in group $i$ at time $t+1$. For example, if $m=3$, equation (1) will be in the form below.

$\left(\begin{array}{l}n_{1}(t+1) \\ n_{2}(t+1) \\ n_{3}(t+1)\end{array}\right)=\left(\begin{array}{ccc}P_{1} & F_{1} & F_{2} \\ G_{1} & P_{2} & 0 \\ 0 & G_{2} & P_{3}\end{array}\right)\left(\begin{array}{l}n_{1}(t) \\ n_{2}(t) \\ n_{3}(t)\end{array}\right)=\left(\begin{array}{c}P_{1} n_{1}(t)+F_{1} n_{2}(t)+F_{2} n_{3}(t) \\ G_{1} n_{1}(t)+P_{2} n_{2}(t) \\ G_{2} n_{2}(t)+P_{3} n_{3}(t)\end{array}\right)$

For $n_{1}(t+1)$, the individuals in age group 1 at time $(t+1)$ consist of the individuals at time $t$ that survive getting older but still in the age group 1 , and individuals at time $t$ that is born from the fertile individuals in age groups 2 and 3. For $n_{2}(t+1)$, the individual in age group 2 at time $(t+1)$ consist of individuals at time $t$ that survive getting older from the age group 1 and 2 . For $n_{3}(t+1)$, the individual in age group 3 at time $(t+$ 1 ) consist of individuals at time $t$ that survive getting older from the age group 2 and 3.

In a group, there are individuals who have been in this group for $1,2, \ldots, d_{i}$ years. Denote $p_{i}$ the probability of an individual in group $i$ surviving to one year ahead. We assume that all individuals within a group are subject to the same survival probability and group duration. The probability of an individual surviving for $d_{i}$ years is $p_{i}^{d i}$, and assume that the survival probability of the youngest individual is 1. Assume that the population is stationary and the age distribution within groups is stable. In the time interval from $t$ to $t+1$, the oldest individuals will transfer, if survive, to the next group, and the younger individuals will remain in the group. The proportion of all remaining individuals at $t+1$ and at time $t$ is following.

$$
P_{i}=\frac{p_{i}+p_{i}^{2}+\cdots+p_{i}^{d_{i}-1}}{1+p_{i}+p_{i}^{2}+\cdots+p_{i}^{d_{i}-1}}=\frac{1-p_{i}^{d_{i}-1}}{1-p_{i}^{d_{i}}} p_{i}
$$

Where each series in numerator and denominator is a geometric series with ratio factor $p_{i}<1$, so its partial summation is $S_{n}=\frac{1-p_{i}^{n+1}}{1-p_{i}}$. The proportion of the oldest individuals survive and the remaining individuals is following.

$$
G_{i}=\frac{p_{i}^{d_{i}}}{1+p_{i}+p_{i}^{2}+\cdots+p_{i}^{d_{i}-1}}=\frac{p_{i}^{d_{i}}\left(1-p_{i}\right)}{1-p_{i}^{d_{i}}}
$$

From equation (1), the number of population at a long time $k$ will depend on the number of initial population or $n(k)=A^{k} n(0)$. An analysis of matrix A is done by calculating its eigenvalues $\lambda_{i}, i=1,2, \ldots, m$ by solving.

$$
|A-\lambda I|=0
$$

Where $I$ is the identity matrix. Consider $\lambda^{\tilde{}}=\max$ $\lambda_{i}$. If $\lambda^{\tilde{n}}=1$ or $\mathrm{A}^{k}$ tends to be an identity matrix, so the whole population is stationary, which means the number of population is constant. If $\lambda^{\tilde{\nu}}>1$ or $\mathrm{A}^{k}$ tends to be infinity matrix, so the population will blow up. The harvesting effort could be done in order to keep the population stable. If $\lambda^{\tilde{<}}<1$ or $\mathrm{A}^{k}$ tends to be a zero matrix, so the population will be diminishing. The annual rate of increase of the population is given by the logarithm of the maximum eigenvalue,

$$
r=\ln \tilde{\lambda}
$$

\subsubsection{Markov Chain Model}

The second model derived in this paper needs Markov Chain model to estimate the long-run behavior. Let $\mathrm{X}_{1}, \mathrm{X}_{2}, \mathrm{X}_{3}, \ldots$ be a sequence of random variables. This sequence has Markov Chain property if

$$
P\left(X_{n+1}=j \mid X_{n}=i, X_{m_{1}}=k_{1}, \ldots, X_{m_{s}}=k_{s}\right), \quad m_{i}<n, i=1,2, \ldots, s
$$

is a probability determined only by $n, i$, and $j$, which is the transition probability from $i$ to $j$ at time $n$. It means that the occurrence of next event at $n+1$ is dependent only on the current event at $n$, but not on events occurred before $n$. If this probability is also independent of any current time $n$, then the Markov Chain is homogeneous and the transition probability is denoted $p_{i j}=P\left(X_{n+1}=j \mid X_{n}=i\right)$. It is obvious that for $\sum_{k=1}^{j} p_{i k}=1$ for all $i$.

Let $\mathrm{X}_{1}, \mathrm{X}_{2}, \mathrm{X}_{3}, \ldots$ be a homogeneous Markov chain, then the s-step transition probabilities are defined for any $\mathrm{k}$ as following.

$$
p_{i j}^{s}=P\left(X_{k+s}=j \mid X_{k}=i\right)
$$

We define $p_{i j}^{s}$ or simplified by $p^{s}$ be $N \times N$ matrix that is called s-step transition matrix. Here $N$ is the number of events or states. $p^{s}$ is $s$-th power of the transition probability matrix. The Chapman - Kolmogorov theorem will be used in determining many steps ahead.

$$
p^{n+m}=p^{n} p^{m}, \quad n, m \geq 0
$$


For long run behavior, the Markov Chain is needed to be in a canonical form. Consider an arbitrary absorbing Markov chain that containing absorbing states, where its probability to be in the same state is 1 , and the transition probability to or from other state is zero. Renumber the states so that the absorbing states come first. If there are $r$ absorbing states and $t$ transient or unabsorbing states, the transition matrix will have the following canonical form.

$$
\widetilde{P}=\left(\begin{array}{c|c}
I & 0 \\
-- & -- \\
R & Q
\end{array}\right)
$$

Where $I$ is an $r \times r$ identity matrix of absorbing states, 0 is an $r \times t$ zero matrix, $R$ is a nonzero $t \times r$ transition matrix from transient states to absorbing states, and $Q$ is an $t \times t$ transition matrix from and to transient states. The first $r$ states are absorbing and the last $t$ states are transient. For 2-steps and 3-steps transition matrices, we obtain respectively.

$$
\widetilde{P}^{(2)}=\left(\begin{array}{cc}
I & 0 \\
R+Q R & Q^{2}
\end{array}\right), \quad \tilde{P}^{(3)}=\left(\begin{array}{cc}
I & 0 \\
R+Q R+Q^{3} R & Q^{3}
\end{array}\right)
$$

Eventually, the n-steps transition matrix is following.

$$
\tilde{P}^{(n)}=\left(\begin{array}{cc}
I & 0 \\
R+Q R+Q^{3} R+\ldots+Q^{n-1} R & Q^{n}
\end{array}\right)
$$

For $n \rightarrow \infty$, it yields $Q^{n} \rightarrow 0$ since the transient probability $p<1$ so $p^{n} \rightarrow 0$. On the other part, if $n \rightarrow \infty$, $\left(1+Q+Q^{3}+Q^{4}+\ldots\right) R=N R$, where $N=(1-Q)^{-1}$ that is called the fundamental matrix. The entry $n_{i j}$ of matrix $N$ is the expected number of times the chain is in state $j$, given that it starts in state $i$. We can have simulation on this entry in order to predict the number of periods for which a desired state of the event is kept in the same state before it transform to the undesired absorbing state.

\section{Results}

Total numbers of trees in years 2011-2015 from teak forest managed by PT Perhutani at Begal, East Java, Indonesia are shown in Table 3. There are 148 sites being observed with various areas from $0.3-84.5$ hectares.

\subsection{Leslie Matrix for the Teak Tree Forest}

Based on the age classification of teak tree described in Section 2, we have time intervals $d_{0}=d_{1}=5, d_{i}=10, i$ $=2,3, \ldots, 6$. The annual survival probability is defined by following formula.

$$
p_{i}=\frac{1}{d_{i}} \sum_{k=1}^{d_{i}-1} \frac{n_{i}(k+1)}{n_{i}(k)}, i=0,1, \ldots, 6
$$

In this research, fertility elements are not considered, $\mathrm{F}_{i}=0$, because the plantation of the new trees is deliberately done by the worker of PT Perhutani. It is assumed that the decreasing of number of trees is only due to normal thinning. The values of $p_{i}$ in (8), $P_{i}$ in (3), and $G_{i}$ in (4) are shown in Table 4. Note that they are unitless values of probabilities.

Its Leslie matrix (2) is following.

$$
A=\left(\begin{array}{ccccccc}
0.7396 & 0 & 0 & 0 & 0 & 0 & 0 \\
0.2334 & 0.7526 & 0 & 0 & 0 & 0 & 0 \\
0 & 0.1408 & 0.8649 & 0 & 0 & 0 & 0 \\
0 & 0 & 0.0661 & 0.8802 & 0 & 0 & 0 \\
0 & 0 & 0 & 0.0789 & 0.8861 & 0 & 0 \\
0 & 0 & 0 & 0 & 0.0842 & 0.8895 & 0 \\
0 & 0 & 0 & 0 & 0 & 0.0881 & 0.8887
\end{array}\right)
$$

The maximum eigenvalue of this matrix is $\lambda^{\tilde{2}}=0.8895<1$ and the annual rate of increase of the population $r$ in (5) is $\ln 0.8895=-0.1171$, so the teak trees population for all age groups is diminishing. See Figure $(2-3)$. It is normal because the plantation of new tree is controlled by the Perhutani staffs. Trees with an appropriate age will be harvested also by them. Using this matrix, the number of teak trees for years in the future can be approximated. We calculate $n_{i}(t$ $+1), i=0,1,2,3,4,5,6$ in (2) iteratively for $t=0,1,2$,

Table 3. Numbers of teak trees at begal

\begin{tabular}{lrrrrr}
\hline $\begin{array}{l}\text { Age } \\
\text { group }\end{array}$ & 2011 & 2012 & 2013 & 2014 & 2015 \\
\hline Nursling & 762923 & 449093 & 228613 & 134598 & 85998 \\
I & 507611 & 761691 & 873816 & 663968 & 631445 \\
II & 88716 & 150332 & 211462 & 265625 & 343980 \\
III & 43870 & 31067 & 31067 & 24920 & 24920 \\
IV & 25078 & 37881 & 36586 & 42112 & 42112 \\
V & 19521 & 19521 & 16272 & 12833 & 12833 \\
VI & 9457 & 9457 & 14001 & 17314 & 17314 \\
\hline
\end{tabular}

Table 4. Probability values for all age groups

\begin{tabular}{llllllll}
\hline & \multicolumn{1}{c}{0} & 1 & \multicolumn{1}{c}{2} & \multicolumn{1}{c}{3} & 4 & 5 & \multicolumn{1}{c}{6} \\
\hline$p_{i}$ & 0.973007 & 0.893426 & 0.930967 & 0.959082 & 0.97069 & 0.977674 & 0.9759 \\
$P_{i}$ & 0.739647 & 0.752593 & 0.864896 & 0.880181 & 0.886092 & 0.889538 & 0.888671 \\
$G_{i}$ & 0.23336 & 0.140833 & 0.066071 & 0.078901 & 0.084598 & 0.088136 & 0.08723 \\
\hline
\end{tabular}


3. We use year 2011 data as the initial value $n_{i}(0)$ and the calculated values $n_{i}(t+1)$ are validated using the exact data $\tilde{n_{i}}(t+1)$ from 2012 - 2015 in Table 3 by calculating relative errors between the approximated and exact data $\tilde{n_{i}}(t), i=0,1,2,3,4,5,6$ using the formula below.

$$
\operatorname{relErr}_{i}(t+1)=\left|\frac{\tilde{n}_{i}(t+1)-n_{i}(t+1)}{\tilde{n}_{i}(t+1)}\right|
$$

The results show that the range of their relative errors is 0.0018-2.5898, which are quite good considering that it is rather uneasy task to collect the data from the resource.

The objective of the reservation management is to make the number of trees of group Nursling $(0-4)$ is decreasing to only $1 \%$ of the initial number (objective 1 ) or to only very small number of trees (objective 2 ), for example it remains only 100 trees. We assume that the planted trees has been grown normally and the forest is well protected. We calculate equation (2) iteratively for $t=0,1,2, \ldots, T^{*}$ such that for the first time $n_{0}\left(T^{*}\right)<0.01 n_{0}(0)$ in objective 1 , or $n_{0}\left(T^{*}\right)<100$ in objective 2 . The calculation results of equation (2) from both objectives is shown in Figure 3.

The calculated time $T^{*}$ for objective 1 is 16 years from 2011. For objective 2 , the calculated time $T^{*}$ is 30 years from 2011. At the end of periods, there will be a good composition of trees' ages, which represents the sustainable condition.

\subsection{Markov Chain Model for Teak Forest}

The first step in deriving model using Markov Chain is the determination of states. We know that the number of teak trees is decreasing due to normal thinning process or illegal logging activities. The last cause that massively done by irresponsible people could give serious problem to the environment's sustainability. We define the Degree of Planting Density (DPD) that is the ratio between the number of trees in the observed area and the standard density for thinning in Table 2. The unit of density is per one hectare. The younger plant has shorter diameter than the older one so the density of younger plants larger than the density of older plants.

We define state 1 (Good Condition) with DPD > 0.6 where there are enough trees at the site. State 2 (Bad Density) is with $0.3<D P D \leq 0.6$ where there are not enough trees at the site. State 3 (Open Area) is $D P D \leq 0.3$ where the site is almost a barren area with barely not more than 60 teak trees on a hectare area are planted.

Now we observe 104 sites of Nursling age group in year 2011 and they are mostly still in this group until 2015. We do not observe for other age groups because all their sites are only in state 1 and 2 so we cannot construct a Markov Chain matrix for these age groups. Except for age group IV (30 - 39), its Markov Chain matrix is an identity matrix that means they are all absorbing states, which is explained later.

The classification of states each year for Nursling age group is shown in Table 5. The number of sites in state 3 in year 2011 increase in year 2012, and then the number is decreasing for other years. The increase is suspiciously due to illegal logging activities at a particular site. So we concern whether this will bad impact in years ahead. The decrease is due to planting activities by PT Perhutani.

The transition probability matrices for (20112012), (2012-2013), (2013-2014), and (2014-2015) are shown as $P_{1}, P_{2}, P_{3}, P_{4}$ in the following matrices.
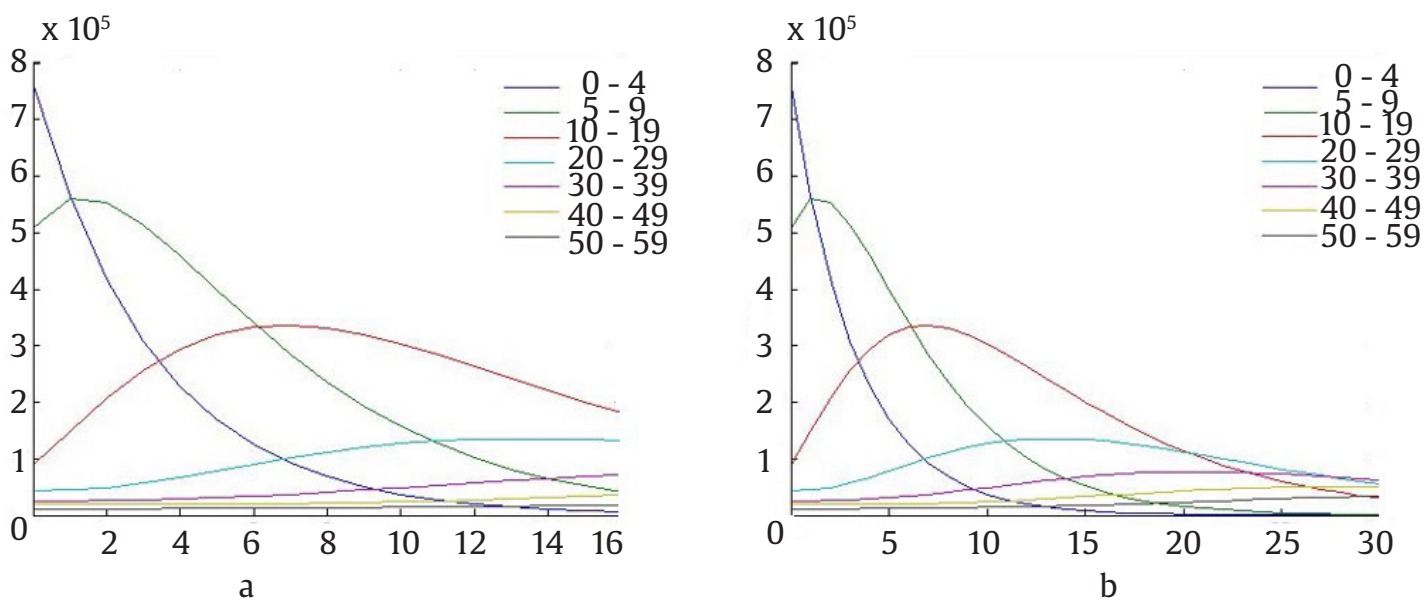

Figure 3. (a) Year 2011 data for group 0 - 4 becomes < 1\% of initial number; (b) Year 2011 data for group 0 - 4 becomes < 100 per hectare 
$P_{1}=\left(\begin{array}{ccc}0.9286 & 0.0714 & 0 \\ 0.0714 & 0.9048 & 0.0238 \\ 0 & 0 & 1\end{array}\right), P_{2}=\left(\begin{array}{ccc}0.9818 & 0.0182 & 0 \\ 0.2143 & 0.7857 & 0 \\ 0 & 0.7143 & 0.2857\end{array}\right)$

$P_{3}=\left(\begin{array}{ccc}0.9206 & 0.0635 & 0.0159 \\ 0.0769 & 0.8718 & 0.0513 \\ 0 & 0.5 & 0.5\end{array}\right), P_{4}=\left(\begin{array}{ccc}1 & 0 & 0 \\ 0.1538 & 0.8462 & 0 \\ 0 & 0 & 1\end{array}\right)$

The entries in the first row of the matrix $P_{k}, k=1,2$, 3 represent the probabilities for the various kinds of states following state 1 (Good Condition). Similarly, the entries in the second and third rows represent the probabilities for the various kinds of states following state 2 (Bad Condition) and state 3 (Open Area), respectively.

In the third row of matrix $P_{1}$, there is probability 1 that means state 3 is absorbing state where the condition is always in this state forever. This case also occurs in the first and third rows of matrix $P_{4}$. The states other than the absorbing state are called transient states, and from any of these it is possible to reach the absorbing states 3 and then to be stuck in state 3 forever.

It also shown all transition matrices have dominant values on the diagonal of the matrices, where the sites are still grow naturally. There are exception on the third row of matrices $P_{2}$ and $P_{3}$, which means there might be plantation activities by by PT Perhutani at the sites with state 3 in those years, so their state changes to state 2 .

In transition matrix $P_{3}$, there is a probability 0.0159 that the sites with state 1 are degraded to state 3 , which means there might be illegal logging or looting activities at that sites.

In order to determine the probability that in 2011 state $i$ occurs and it will be in state $j$ two years later, the 2-steps matrix from 2011 to 2013 is obtained by the following formulas.

$$
\begin{aligned}
p_{i j}^{(2)}=P\left(X_{3}=\right. & \left.j \mid X_{1}=i\right) \\
& =P\left(X_{3}=j \mid X_{1}=i, X_{2}=1\right) P\left(X_{2}=1 \mid X_{1}=i\right) \\
& +P\left(X_{3}=j \mid X_{1}=i, X_{2}=2\right) P\left(X_{2}=2 \mid X_{1}=i\right) \\
& +P\left(X_{3}=j \mid X_{1}=i, X_{2}=3\right) P\left(X_{2}=3 \mid X_{1}=i\right)
\end{aligned}
$$

For all $i, j=1,2,3$, the resulted matrix $P^{(2)}$ can be obtained by doing the matrix multiplication $P_{1}$ and $P_{2}$.

Table 5. The number of sites for each state

\begin{tabular}{cccc}
\hline Year & State 1 & State 2 & State 3 \\
\hline 2011 & 56 & 42 & 6 \\
2012 & 55 & 42 & 7 \\
2013 & 63 & 39 & 2 \\
2014 & 62 & 39 & 3 \\
\hline
\end{tabular}

$$
P^{(2)}=\left(\begin{array}{lll}
p_{11}^{(2)} & p_{12}^{(2)} & p_{13}^{(2)} \\
p_{21}^{(2)} & p_{22}^{(2)} & p_{23}^{(2)} \\
p_{31}^{(2)} & p_{32}^{(2)} & p_{33}^{(2)}
\end{array}\right)=P_{1} P_{2}
$$

For example, $p_{11}^{(2)}=0.9286 \times 0.9818+0.0714 \times$ $0.2143=0.9270$. The 3 -steps matrix from 2011 to 2014 is obtained by similar procedures.

Using Chapman - Kolmogorov theorem, the transition probability matrices for more than one year can be approximated by using the multiplications of transition matrix 2011. For example,

$$
\begin{aligned}
& p_{11}^{(2)}=p_{11} p_{11}+p_{12} p_{21}+p_{13} p_{31}, \\
& p_{12}^{(2)}=p_{11} p_{12}+p_{12} p_{22}+p_{13} p_{31}, \\
& p_{13}^{(2)}=p_{11} p_{13}+p_{12} p_{23}+p_{13} p_{31},
\end{aligned}
$$

which are the elements on the first row of matrix $P_{1}^{2}$.

$$
P_{1}^{2}=P_{1} P_{1}=\left(\begin{array}{ccc}
0.8673 & 0.1310 & 0.0017 \\
0.1310 & 0.8237 & 0.0454 \\
0 & 0 & 1
\end{array}\right)
$$

Having validated this approximation, the differences or errors between elements of $P_{1}^{2}$ and $P_{1} P_{2}$ are in range $0.0017-0.7143$.

Now we do simulation on data so the condition of teak forest in Begal for long run can be estimated. There are 3 scenarios being exercised, the first one is based on the existing data, and the other two are simulations by doing a small change from the existing data deliberately on particular sites in order to obtain desired objectives in the long-run.

\subsubsection{First Scenario}

Certain changes of states obtained from data in years 2011 - 2012 are shown in Table 6.

Matrix $\tilde{\mathrm{P}}$, which is the canonical form of matrix $\mathrm{P}_{1}$, and its fundamental matrix are following.

$$
\tilde{P}=\left(\begin{array}{lll}
p_{33} & p_{31} & p_{32} \\
p_{13} & p_{11} & p_{12} \\
p_{23} & p_{21} & p_{22}
\end{array}\right)=\left(\begin{array}{ccc}
1 & 0 & 0 \\
0 & 0.9286 & 0.0714 \\
0.0238 & 0.0714 & 0.9048
\end{array}\right), N=\left(\begin{array}{ll}
56 & 42 \\
42 & 42
\end{array}\right)
$$

Table 6. States changes of original data

\begin{tabular}{clc}
\hline State Change & \multicolumn{1}{c}{ Site names } & Number of sites \\
\hline $1 \rightarrow 2$ & 10D, 18G, 33B, 34A & 4 \\
$2 \rightarrow 1$ & 26C, 27F, 32A & 3 \\
$2 \rightarrow 3$ & $9 \mathrm{~F}$ & 1
\end{tabular}


From the fundamental matrix, $n_{11}=56$ is the number of years needed that state 1 being kept in the same state before being absorbed. Note that the harvesting time for teak tree is 60 years, so there is a possibility on which the transition from state 1 to state 3 will occur before the harvest time. That will be a big loss to PT Perhutani, so we can claim that the current forest condition is critical.

\subsubsection{Second Scenario}

If site $10 \mathrm{~B}$, which is in state 2 for both years, is to be maintained by full monitoring program in order to be upgraded to state 1 in year 2012. The transition probability matrix is then recalculated.

Its canonical matrix $\tilde{P}$ and fundamental matrix $N$ are following.

From the fundamental matrix, $n_{11}=70>60$ is the time needed that state 1 being in the same state before being absorbed. It is enough time to harvest the wood before it will transformed to be open areas with small number of trees.

\subsubsection{Third Scenario}

If site $10 \mathrm{D}$, which is in state 1 in 2011, is to be maintained by full monitoring in order to avoid illegal logging or looting so it keeps to be in state 1 . The transition probability matrix is recalculated.

Its canonical matrix $\mathrm{P}^{\sim}$ and fundamental matrix $N$ are following.

$$
P=\left(\begin{array}{ccc}
1 & 0 & 0 \\
0 & 0.9464 & 0.0536 \\
0.0238 & 0.0714 & 0.9028
\end{array}\right), N=\left(\begin{array}{ll}
74.6269 & 40.0168 \\
55.9701 & 40.0168
\end{array}\right)
$$

From the fundamental matrix, $n_{11}=74.6269>60$ is the time needed that state 1 being in the same state before being absorbed. It is enough time to harvest the wood before it will transformed to be open areas with small number of trees.

Another attempt to upgrade site $9 \mathrm{~F}$ in order to be kept in state 2, not degrading as in the data. However, the matrix $Q$ will be singular so its matrix fundamental cannot be obtained.

\section{Discussion}

We compare these factors below for both developed models.

1. Usefulness

Leslie Matrix model could predict the period of time needed from year 2011 for the forest to
Table 7. States changes with modified site 10B

\begin{tabular}{clc}
\hline State change & \multicolumn{1}{c}{ Sites } & Number of sites \\
\hline $1-2$ & 10D, 18G, 33B, 34A & 4 \\
$2-1$ & 26C, 27F, 32A, 10B & 4 \\
$2-3$ & 9F & 1 \\
\hline
\end{tabular}

Table 8. States changes with modified site 10D

\begin{tabular}{clc} 
State change & \multicolumn{1}{c}{ Sites } & Number of sites \\
\hline $1-2$ & 18G, 33B, 34A & 3 \\
$2-1$ & 26C, 27F, 32A & 3 \\
$2-3$ & $9 \mathrm{~F}$ & 1 \\
\hline
\end{tabular}

achieve sustainable condition that there will be a good composition of the trees' ages. Markov Chain model could propose improvement on managing the sites so the teak trees can be lasting until the harvest time.

2. Input of the model

Leslie Matrix model needs classification of age groups of teak trees and the numbers of trees for each group. Markov Chain model needs determination of DPD (Degree of Planting Density) per hectare of areas.

3. Advantages

Leslie Matrix model can be implemented into data of any forest because it is constructed from the Table of Standard Density for Thinning made from good practices on managing the teak tree forest. In Markov Chain, the transition probability matrix can be constructed easily based on the desired change of DPD (Degree of Planting Density) on a certain age group.

4. Disadvantages

Leslie Matrix model has assumption that there is no extreme illegal logging and looting activities along the period of time. In constructing Markov Chain matrix, all determined states and transition between states should occur in the observed age group. If not, the matrix will be not existing or singular.

To conclude, both models have advantages in predicting the condition of the teak tree forest in the future. The results show a quite critical condition in current state. The first model using Leslie Matrix shows that the current condition is not guaranteed to sustain because it needs 16 years from year 2011 that the sustainable condition of the forest can be achieved. The second model using Markov Chain shows that the good condition part based on its density of the early age group ( 0 - 4 years) has potential to become the worst condition before its harvest time. Some effort are needed to be done in order to repair the current 
condition. However, models developed in this paper still have not very small errors in the validation process because the recorded data is not enough and updated. If the data is available in enough number, we could choose the appropriate initial values that represent the real condition of the teak forest.

\section{Acknowledgements}

The travel grant of this research is supported by 2017 P3MI - ITB Research Grant.

\section{References}

Caswell H. 2001. Matrix Population Models: Construction, analysis, and interpretation. $2^{\text {nd }}$ Edition.

Crouse et al. 1987. A Stage-based Population Model for Loggerhead Sea Turtles and Implications for Conversation, Ecology.

D'Arrigo $\mathrm{R}$ et al. 2011. Three centuries of Myanmar monsoon climate variability inferred from teak tree rings. Geophysical Research Letters 38:24705. doi:10.1029/2011GL049927

"GRIN Taxonomy for Plants- Tectona". United States Department of Agriculture. 5 October 2007. Retrieved 24 September 2017.

Kemeny GJ and Snell JL. 1976. Finite Markov Chain. New York: Springer-Verlag.

Kollert W and Cherubini L. 2012. Teak Resources and Market Assessment 2010 (Tectona grandis Linn. F.). Planted Forests and Trees Working Paper Series. Food and Agriculture Organization of the United Nations.
Leslie PH. 1948. Some Further Notes on the Use of Matrices in Population Mathematics. Biometrika 35:213-245

Nunifu KT. 1997. The growth and yield of teak (Tectona grandis Linn F.) plantations in Northem Ghana. MSc Thesis, Faculty of Forestry, Lakehead University.

Ram S et al. 2008. Tree-ring analysis of teak (Tectona grandis L.F.) in central India and its relationship with rainfall and moisture index. Journal of Earth System Science 117:637-645.

Shamaki SB et al. 2016. Height-diameter Relationship Models for Teak (Tectona grandis) Plantation in Nimbia Forest Reserve, Nigeria. Asian Journal of Environment and Ecology 1: XX-XX.

Sheldon RM. 2010. Introduction to Probability Models, $10^{\text {th }}$ Edition. Academic Press.

Smith GC and Trout RC. 1994. Using Leslie Matrices to Determine Wild Rabbit Population Growth and the Potential for Control. Journal of Applied Ecology 31:223-230.

Tewari VP et al. 2014. Developing a dynamic growth model for teak plantations in India. Forest Ecosystems 1:9. http://www.forestecosyst.com/content/1/1/9

https://commons.wikimedia.org/wiki/File:Redwood_ National_Park,_fog_in_the_forest.jpg accessed on 30th September 2017.

Buku Prosedur Kerja (Book of Working Procedure), PT Perhutani, Indonesia (without explicit publishing year). 